\section{CONTROL OF RADIAL} INCREMENT AND WINDING DENSITY OF COMPOSITE CYLINDRICAL SHELLS

ALEX KUTIN ${ }^{1}$, GENNADY ARYASSOV ${ }^{2}$, VICTOR MUSALIMOV ${ }^{3}$, TRIEU MINH VU' ${ }^{2}$, REZA MOEZZI ${ }^{4,5}$, JINDRICH CYRUS ${ }^{5}$

${ }^{1}$ Society with Limited Liability "VP PETRO IN TRADE", SaintPetersburg, Russia

${ }^{2}$ School of Engineering, Tallinn University of Technology, Tallinn, Estonia

${ }^{3}$ Department of Mechatronics, National Research University of Information Technology, Mechanics and Optics (ITMO),

Saint-Petersburg, Russia

${ }^{4}$ Faculty of Mechatronics, Informatics and Interdisciplinary Studies, Technical University of Liberec, Liberec, Czech

Republic

Institute for Nanomaterials, Advanced Technologies and Innovation, Technical University of Liberec, Liberec, Czech

Republic

DOI : 10.17973/MMSJ.2020_11_2020058

Reza.Moezzi@tul.cz

There is an effective method of filament winding for the manufacture of hollow composite products (pipes, containers, etc.). Finished products are light, durable and widely used in industry. However, this method has significant drawbacks. The tension of the surface layer and the tension of the inner layers can differ significantly for the products being formed. This effect is also observed when the tension of the wound material is adjusted. Another significant drawback of the technology is the change in the diameter of the cross section of the product in the end zones, where the return movement of the movable trolley with threads (tape) is carried out. As a result of these drawbacks, there are deviations in the winding density and other stressstrain state indicators from the planned values. To eliminate these shortcomings, we propose an original method for controlling the radial increment of the composite winding and its density.

KEYWORDS

Composite material, winding, thread tension, winding process control, adaptive control

\section{INTRODUCTION}

Relatively all pipes and industrial containers have been made by metals in recent and current century. Nowadays composite materials are used for their production. One of the well-known and effective methods of manufacturing hollow products (pipes and large containers) is filament winding. It is carried out by special devices which can be also designed in a small scale for research purposes [Saad Mutasher 2012]. The fibers are wound from the bobbins mounted on the stand, soaked in a special bath with resin to strengthen their structure and then glued in the winding, then wound on a rotating mandrel.

In addition to the noticeable advantages of filament winding, this method has some important drawbacks. For example, despite adjusting the tension of the feed material in order to maintain it at a given level [Li 2015], [Shengle 2009], [Zhang 2017], [Srivastava 2016] the tension of the surface layer and the inner layers cannot be stabilized almost for all reproducible products, for ensuring the same values of the winding radius and its density [Musalimov 2016] (in Russian). The reason for this phenomenon is the heterogeneity of the wound material in terms of physical and mechanical parameters (linear density, characteristics of elastic properties, humidity, etc.) [Obraztsov 1986] (in Russian), [Thwaits 1977] and the random nature of the actual winding process. Changing any technological factor associated with the winding process and affecting it inevitably leads to a deviation of the winding point movement from the planned trajectory. Another significant drawback of the technology is the change in the diameter of the cross section of the product in the end zones, where the return movement of the mobile trolley with threads (tape) is carried out.

Therefore, the stabilization of the winding density of a composite product is a vital task and this novel paper proposes to accomplish mentioned task by controlling the winding process in a composite cylindrical shell. Current studied of solving this problem [Hashimoto 2016], [Hashimoto 2010] are not effective enough due to the lack of relationship between the geometric parameters of the created winding and the parameters of its stress state.

\section{FORMATION MODEL OF THE COMPOSITE WINDING AND DETERMINATION OF THE KINEMATIC PARAMETERS}

To solve this problem, we have proposed a device for controlling the process of forming the winding, developed based on the model of its formation [Kutin 2018] (in Russian):

$$
\begin{aligned}
A & =f(\theta), \rho(\theta)-\rho_{0}=\int_{0}^{\theta} a d \theta, L=\int_{0}^{\theta} \rho(\theta) d \theta, \\
\gamma & =\frac{m_{S} D(\rho)}{\pi H\left(\rho^{2}-\rho_{0}^{2}\right)}
\end{aligned}
$$

where $\theta$ is the angle of rotation of the mandrel, a is the parameter of the winding spiral, $\rho_{0}$ is the radius of the mandrel, $m_{\mathrm{S}}$ is mass of the unit of length of the wound material, $\mathrm{H}$ is the length of the mandrel, $\rho$ is the current radius of the winding, $L$ is the length of the composite layer of wound material and $D$ is the length of the wound material.

The parameter of the winding spiral is determined by the ratio

$a=\frac{v}{\omega}=\frac{d \rho}{d \theta}=f(\theta)$

where $u$ is the velocity of the winding point (the contact point of the wound material with the wound body) in the radial direction $(u=d \rho / d t), \omega$ is angular velocity of the mandrel $(\omega=$ $d \theta / d t), t$ is the current time of movement of the winding point

With a small wound thickness, the parameter a does not change its value during the winding process therefore, we can consider a =const, and the current winding radius is calculated using the Archimedean spiral [Kutin 2018] (in Russian):

$\rho(\theta)=\rho_{0}+a \theta$

and the length of the composite layer of the wound material is calculated using a simplified formula for straightening the spiral arc [Kutin 2004]:

$L=\frac{a \theta^{2}}{2}+\rho_{0} \theta=\frac{\rho^{2}-\rho_{0}^{2}}{2 a}$.

Equation (4) relates the motion of the winding point $p(\theta)$ in a projection on a plane perpendicular to the axis of rotation to the length of the composite layer $L(\theta)$. According to equation (1), to determine the winding density $\gamma(\theta)$, this is not enough, and it is necessary to calculate the length of the wound material $D(\rho)$. To do this, we must consider the movement of the winding point on the mandrel surface in a cylindrical coordinate system. In a 
cylindrical coordinate system, the position of the winding point $\vec{r}$ at time $t$, in addition to the coordinates $p, \theta$, will be determined by the coordinate $z(r=\{p, \theta, z\})$, which sets the value of the segment on the forming cylindrical surface from the plane perpendicular to the axis of rotation in which $p$ and $\theta$ are deposited, to the winding point.

The range of changing the $z$ coordinate is limited by the length of the mandrel $(0 \leq z \leq H)$. In this range, the movement of the winding point along the forming cylindrical surface is determined by the movement of the spreader in the end zones and in the regular zone $\left(\mathrm{H}=\mathrm{H}_{T}+\mathrm{H}_{\mathrm{P}}+\mathrm{H}_{T}\right)$. In the end zone $\mathrm{H}_{T}$ of the shell, where the laying device reverses, the winding is carried out along the line of equal geodesic deviation [Komkov 2012]. In this zone, there is a smooth change in the angle of winding $\beta_{\text {T }}$ of the material on the shell from the calculated value $\beta$ to the limit value $\beta_{L}=\pi / 2$ when the velocity $V_{T}$ of the laying device changes from the value $V$ to 0 or vice versa. In the regular zone $H_{p}$ the velocity of the laying device is constant $\left(\mathrm{V}_{\mathrm{T}}=\mathrm{V}=\right.$ const $)$, so

$r=\left\{\rho, \theta, H_{T}+\frac{H_{T}}{2}\left[1-(-1)^{n}\right]+(-1)^{n} V \cdot t\right\}$ at

$\mathrm{H}_{\mathrm{T}}<\mathrm{z}<\mathrm{H}_{\mathrm{T}}+\mathrm{H}_{\mathrm{p}}$

where $n$ is the number of laying device reverses $(n=0,1,2,3 \ldots)$. When it is impossible to neglect the width $g$ of the wound material or group of threads, the $z$ coordinate is the coordinate of the point lying in the middle of the place occupied by the wound material in the zone of its contact with the wound body, on the forming cylindrical surface. In this case, the changing range of the $z$ coordinate will be reduced by the value $g(0 \leq z \leq$ $\mathrm{H}-\mathrm{g}$ ), since the range of thread layout in the end zones of the mandrel will be reduced $H_{T}^{*}\left(H_{T}^{*}=\mathrm{H}_{\mathrm{T}}-\mathrm{g} / 2\right)$.

In the end zones the velocity $V_{T}$ of the laying device varies from 0 to $V$ and vice versa. Considering the small size of these zones in relation to the regular zone, the movement of the winding point in them can be approximated equally slow or equally accelerated, then:

$\mathrm{r}=\left\{\rho, \theta, \frac{\mathrm{H}_{\mathrm{T}}}{2}\left[1-(-1)^{\mathrm{n}}\right]+(-1)^{\mathrm{n}} \mathrm{V} \cdot \frac{\mathrm{t}}{2}\right\}$

at $0 \leq \mathrm{z} \leq \mathrm{H}_{\mathrm{T}}$

$$
\mathrm{r}=\left\{\rho, \theta,\left(\mathrm{H}_{\mathrm{T}}+\mathrm{H}_{\mathrm{P}}\right)+\frac{\mathrm{H}_{\mathrm{T}}}{2}\left[1-(-1)^{\mathrm{n}}\right]+(-1)^{\mathrm{n}} \mathrm{V} \cdot \frac{\mathrm{t}}{2}\right\} \text { at }
$$

$\mathrm{H}_{\mathrm{T}}+\mathrm{H}_{\mathrm{P}} \leq \mathrm{z} \leq \mathrm{H}$

The current value of the angle of winding $\beta_{\text {T }}$ of the material on the shell are in these zones, where the laying of turns is carried out along the line of equal geodetic deviation, depends on the angle of geodetic deviation $\phi$, which characterizes the equilibrium of the thread (fiber) on the surface of the mandrel. The tangent of this angle is the ratio of geodesic curvature to normal [Komkov 2012]:

$\tan \varphi=K_{g d} / K_{n}=R_{n} / R_{g d}$

where $K_{n}$ and $K_{g d}, R_{n}$ and $R_{g d}$ are normal and geodesic curvatures of a turn and their radius. The normal curvature is determined from the Euler equation:

$$
K_{n}=K_{1} \cos ^{2} \beta_{\mathrm{T}}+K_{2} \sin ^{2} \beta_{\mathrm{T}},
$$

where $K_{1}$ and $K_{2}$ are the largest and smallest normal curvature of the surface at the point of winding. In this case, for a line of equal geodesic deviation $K_{1}=0$ and $K_{2}=1 / \rho$.

From equation (8), considering (9), it follows:

$$
R_{g d} \tan \varphi=\rho / \sin ^{2} \beta_{\mathrm{T}} .
$$

Radius $R_{g d}$ of the geodesic curvature of the coil for the unfolding cylindrical surface of the mandrel (Fig. 1) can be calculated from:

$$
R_{g d}=d s / d \beta_{\mathrm{T}}=d z /\left(d \beta_{\mathrm{T}} \cos \beta_{\mathrm{T}}\right) .
$$

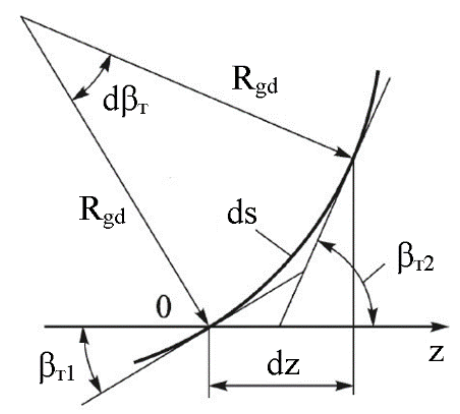

Figure 1. Radius of geodesic curvature of the turn

After substituting (10) into (11), we get:

$$
d z=\frac{\rho}{\tan \varphi} \frac{\cos \beta_{\mathrm{T}}}{\sin ^{2} \beta_{\mathrm{T}}} d \beta_{\mathrm{T}}
$$

Integrating the expression (12) allows us to find the dependence of the winding angle $\beta_{\text {T }}$ on the value of the movement of the winding point along the $\mathrm{z}$ coordinate axis in the end zones when $\mathrm{Z}$ changes from 0 to $\mathrm{H}_{\mathrm{T}}$ or from $\mathrm{H}$ to $\mathrm{H}_{\mathrm{T}}+\mathrm{H}_{\mathrm{p}}$ :

$$
t \frac{1}{\sin \beta_{\mathrm{T}}}=1+\left|\left[z-\frac{H}{2}\left(1-(-1)^{n}\right)\right] \frac{\tan \varphi}{\rho}\right| .
$$

The modulus of the summand in (13) is caused by a change in the direction of movement of the thread laying device and, accordingly, a change in the direction of reference point of the winding angle.

Similarly, the dependence of the winding angle $\beta_{T}$ when changing $\mathrm{z}$ from $\mathrm{H}_{\mathrm{T}}+\mathrm{H}_{\mathrm{p}}$ to $\mathrm{H}$ or from $\mathrm{H}_{\mathrm{T}}$ to 0 is determined:

$\frac{1}{\sin \beta_{\mathrm{T}}}=\frac{1}{\sin \beta}-\left[\frac{\left.H_{T}+H_{P}\right)}{2}\left(1-(-1)^{n+1}\right)+(-1)^{n+1} z\right] \frac{\tan \varphi}{\rho}$

The current value of the winding angle $\beta_{\mathrm{T}}$ is related to the angle of rotation of the mandrel in the end zone $\theta \tau$ by a differential ratio (see Fig.1 [Komkov 2012]) :

$$
\tan \beta_{\mathrm{T}}=\frac{\rho d \theta_{\tau}}{d z}, \quad d \theta_{\tau}=\frac{d z \tan \beta_{\mathrm{T}}}{\rho} .
$$

After substituting (12) into (15) and integrating, we get:

$$
\theta_{\tau}=\frac{1}{\tan \varphi}\left[\ln \left(\tan \frac{\beta_{\mathrm{T}}}{2}\right)-\ln \left(\tan \frac{\beta}{2}\right)\right] \text {. }
$$

When the end zone is completely passed by the laying device the mandrel will turn at an angle in accordance with (16):

$$
\theta_{r}=\frac{1}{\tan \varphi} \ln \left(\frac{\sin \beta}{1-\cos \beta}\right) \text {. }
$$

The angle of winding the material on the shell $\beta T$ in the regular zone is determined by the velocity of the laying device and reaches the value $\beta$ (Fig. 2). It can be calculated based on the ratio of the distance $h$ passed by the laying device during $t_{1}$ of one turn of the mandrel ( $t_{1}=2 \pi / \omega$ at $\omega=$ const) to the value of the winding circle length $\beta$ :

$h=V \cdot t_{1}=V \frac{2 \pi}{\omega}, \quad \beta=\operatorname{arcctg}\left(\frac{V}{\rho \cdot \omega}\right)$, 


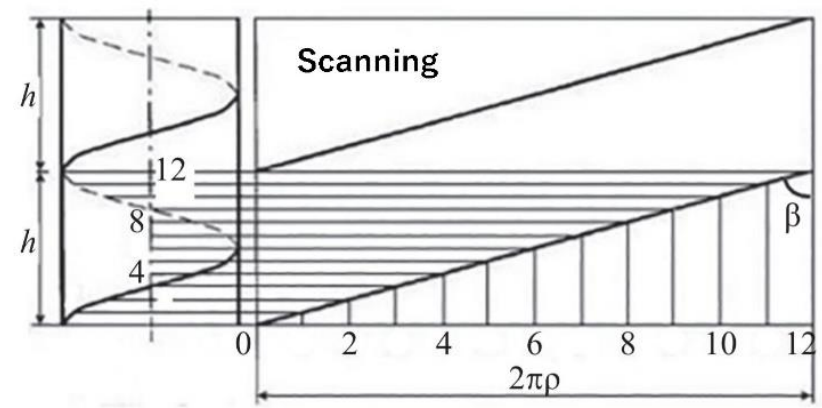

Figure 2. Scanning a thread loop on a cylindrical surface

The choice of the approximating function describing the motion of the winding point in the end area, the relationships describing the movement as equally slow (equally accelerated), allows you to calculate the time of passing the end zone by the laying device $\mathrm{H}_{\mathrm{T}}$ and the $\mathrm{y}$ position of the winding point on it:

$$
t_{\text {rev }}=\frac{2 H_{\mathrm{T}}}{V}, \quad y=\frac{t_{\tau} \cdot V}{2}, \quad z(y)=(-1)^{n} y+\frac{H}{2}\left(1-(-1)^{n}\right)
$$

where $t_{\tau}$ - current time of movement of the laying device in the end zone, $t \tau \in[0, \tau]$.

Taking into account $\beta_{T}$ in (13) or (14) and $z$ in (19), equation (16) is a function $\theta_{\tau}$, depending on the time $t_{\tau}$, according to which the angular velocity in the end zone is determined:

$$
\omega_{\tau}=\frac{\theta_{\tau}\left(t_{\tau}\right)}{t_{\tau}}
$$

The last expression is necessary for generating control actions on the mandrel drive in order to regulate its angular velocity when the winding point moves in the end zones during the formation of a composite product. Thus, the dependencies $(13,14,16,19,20)$ allow us to make the necessary and correct changes to the algorithm for controlling the winding process [Kutin 2018] and increase its efficiency.

The value of the length of the end zone $\mathrm{H}_{T}$ of the shell, where the laying device reverses, according to (13) and (14) is associated with the angle of geodesic deviation $\phi$ by the formula [Komkov 2012]:

$$
H_{\mathrm{T}}=\frac{\rho}{\tan \varphi}\left(\frac{1-\sin \beta}{\sin \beta}\right), \quad \tan \varphi=\frac{\rho}{H_{\mathrm{T}}}\left(\frac{1-\sin \beta}{\sin \beta}\right) .
$$

The length of the wound material $D$ is calculated according to the movement of the laying device. The distance $h$ passed by the laying device in the regular zone during $t_{1}$ of one rotation of the mandrel $\left(t_{1}=2 \pi / \omega\right.$ at $\omega=$ const) is calculated using the formula (18). The velocity of the laying device determines the winding angle $\beta$ (Fig. 2). At this angle $\beta$, the material to be wound is fed in the so-called regular zone HP of the cylindrical shell, where the velocity of the laying device is constant $(\mathrm{V}=$ const). As shown in the scan (Fig. 2), the length of one turn of wound threads in this zone is determined by the expression:

$$
D_{\mathrm{B}}=\sqrt{(2 \pi \rho(\theta))^{2}+h^{2}}=\sqrt{(2 \pi \rho(\theta))^{2}+\left(\frac{2 \pi V}{\omega}\right)^{2}} .
$$

With this in mind, the length of the coiled threads in the regular zone $H_{p}$ of the mandrel is calculated as follows:

$H \mathrm{p} / h \cdot D_{\mathrm{B}}=\frac{H_{p} \omega}{2 \pi V} \sqrt{(2 \pi \rho(\theta))^{2}+\left(\frac{2 \pi V}{\omega}\right)^{2}}$,

where $H_{p} / h$ is the number of revolutions made by the mandrel when winding in the regular zone $\mathrm{H}_{\mathrm{p}}$.

In the end zone $H_{T}$, where the laying device reverses, the length of the wound material $\mathrm{s}$ is determined based on the previously specified differential ratio (12) and the ratio that occurs in the curved triangle (see Fig. 1): $d z=d s \cos \beta_{\mathrm{T}}$.

After substituting (24) into (12), we get:

$d s=\frac{\rho}{\tan \varphi \sin ^{2} \beta_{\mathrm{T}}} d \beta_{\mathrm{T}}$

Integrating, we get:

$s=\frac{\rho}{\tan \varphi} \int_{\beta}^{\pi / 2} \frac{1}{\sin ^{2} \beta_{\mathrm{T}}} d \beta_{\mathrm{T}}=\left.\frac{\rho}{\tan \varphi}\left(-\cot \beta_{\mathrm{T}}\right)\right|_{\beta} ^{\frac{\pi}{2}}=\frac{\rho}{\tan \varphi} \operatorname{ctan} \beta$.

(26)

Taking into account (18) the length of the threads in the reverse zone is:

$s=\frac{\rho}{\tan \varphi} \frac{V}{\rho \omega}=\frac{V}{\omega \tan \varphi}$.

Adding (23) and (27), we find the length of the material that is wound in one pass of the laying device:

$$
\lambda_{c}=\frac{H_{p} \omega}{2 \pi V} \sqrt{(2 \pi \rho(\theta))^{2}+\left(\frac{2 \pi V}{\omega}\right)^{2}}+\frac{2 V}{\omega \tan \varphi} .
$$

However, it should be noted that the calculation of the winding length, taking into account the presence of the mandrel rotation sensor in the control device [Kutin 2018], should be carried out using the number of revolutions made by the packing as an argument. In this case, the integral for (25) is the following expression:

$s_{\mathrm{T}}=\frac{\rho}{\tan \varphi} \int_{\beta_{\mathrm{T}}}^{\pi / 2} \frac{1}{\sin ^{2} \beta_{\mathrm{T}}} d \beta_{\mathrm{T}}=\left.\frac{\rho}{\tan \varphi}\left(-\operatorname{ctg} \beta_{\mathrm{T}}\right)\right|_{\beta_{\mathrm{T}}} ^{\frac{\pi}{2}}=$ $\frac{\rho}{\tan \varphi} \operatorname{ctan} \beta_{\mathrm{T}}$,

or, considering (16):

$$
s_{\mathrm{T}}=\frac{\rho}{\tan \varphi} \operatorname{ctan} \beta_{\mathrm{T}}\left(\theta_{\tau}\right),
$$

where $\theta_{\tau}(\theta)$ is sequentially adding elements of the material length obtained according to (23) or (30), we calculate its total value:

$$
D_{k}=D_{k-1}+
$$

$\int \frac{\rho}{\operatorname{tg} \varphi}\left(\cot \beta_{\mathrm{T}}(\theta)+C_{\Delta \beta_{\mathrm{T}}}\right)$, if the winding point is in the end zone, $2 \pi \Delta \theta \sqrt{(2 \pi \rho(\theta))^{2}+\left(\frac{2 \pi \mathrm{V}}{\omega}\right)^{2}}$, if in the regular zone,

where $\Delta \theta$ is increment of $\theta$ since the calculation of the penultimate value Dk-1 of the length of the wound material, $\Delta \beta_{\mathrm{T}}$ - increment of $\beta_{\mathrm{T}}, C_{\Delta \beta_{\mathrm{T}}}$ - integration constant (25), determined by the increment value $\Delta \beta_{\mathrm{T}}$ of the winding angle, $\rho(\theta)$ we consider it a constant value when the material (thread) is moving until the direction changes.

Using (31), where $D(\rho)=D k$, the winding density $\gamma(\theta)$ is calculated according to (1). As the analysis of expression (1) shows, to form a composite winding with equal technological parameters (radius, length, density), it is necessary to maintain the actual velocity of movement of the winding point equal to the theoretical one. In practice, this means that you need to maintain the actual radius of the formed winding equal to the theoretical one for each current value of the rotation angle $\theta$ of the mandrel. The value of the deviation of the actual winding radius from the theoretical radius at this value of the rotation angle should be used to build a control system for the winding formation process. It is necessary to influence the winding with a variable force [Hashimoto 2016], [Hashimoto 2010], adequately responding to all random disturbances and ensuring equality between the actual and theoretical winding radius. 


\section{CONTROL DESIGN}

Some robotics-based control devices had been studied in the last decade [Weifeng 2013], [Quanjin 2018], [Sorrentino 2017], but in this paper a novel control device was designed to implement the proposed method as shown in Fig. 3.

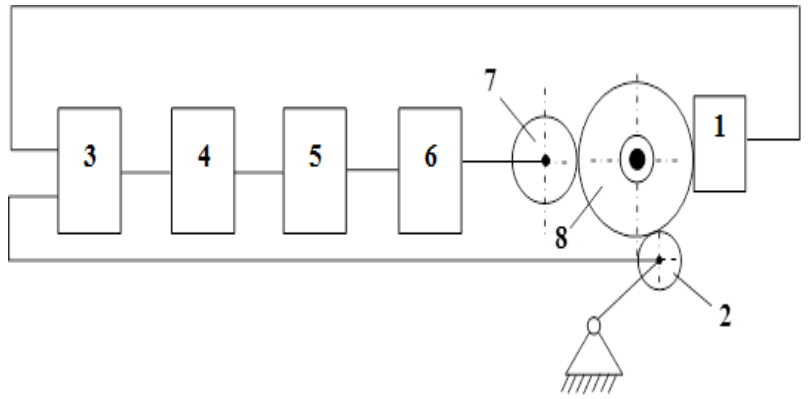

Figure 3. Block diagram of the control unit for monitoring the winding layer formation

The device includes a mandrel rotation sensor 1 , a winding radius sensor 2 , a winding formation control unit 3 , a digital-toanalog converter 4 , a matching device 5 , an electromagnetic

coupling 6 , a sealing body 7 , and a mandrel 8 . In the permanent storage device of the control unit 3, constant values are entered: the values of the radius $\rho 0$ of the mandrel, its width $H$, and the value $r$ of the nominal theoretical increment of the winding radius. Based on the initial data, the coefficient $b=M T /(\pi \mathrm{H})$ is calculated, the value of which is entered into the random-access memory (RAM) of block 3 . In addition, block 3 calculates the final number of turns of the mandrel $n_{k}$ and the number of turns $K_{2}$ of the mandrel through which the pressure roller must move by an amount equal to $r$.

Any PC-compatible industrial controllers, such as the Adam-5000 series controllers manufactured by Advantech or similar from other manufacturers, can be used as the control unit 3. During winding, the sensors measure the actual winding radius, the value of which is recorded in the memory cell of the computing device. For the given number of mandrels turns, the theoretical winding radius is calculated in accordance with the mathematical model. When the computing device counts the specified number of turns of the mandrel, the sealing body is in a fixed position, that is, it is not withdrawn, which allows you to quickly achieve the cylindrical winding. After calculating the set number of turns of the mandrel and calculating the theoretical winding radius, its value is compared with the actual radius. The information obtained as a result of the comparison allows the computing device to determine the value of the diversion of the sealing body of the winding unit and issue a signal to the Executive mechanism for the forced diversion in order to ensure equality between the specified radii. When the number of turns of the mandrel reaches the final set value $n_{k}$, the winding process is completed.

The MATLAB application package was used to simulate the process of controlling the density of composite winding. The MATLAB app allows you to analyze device performance and data on each of its individual knot. Figure 4 shows the control process model built in "Simulink». The simulation results are shown in Figure 5. In particular, the change in the actual winding density during the winding process is shown.

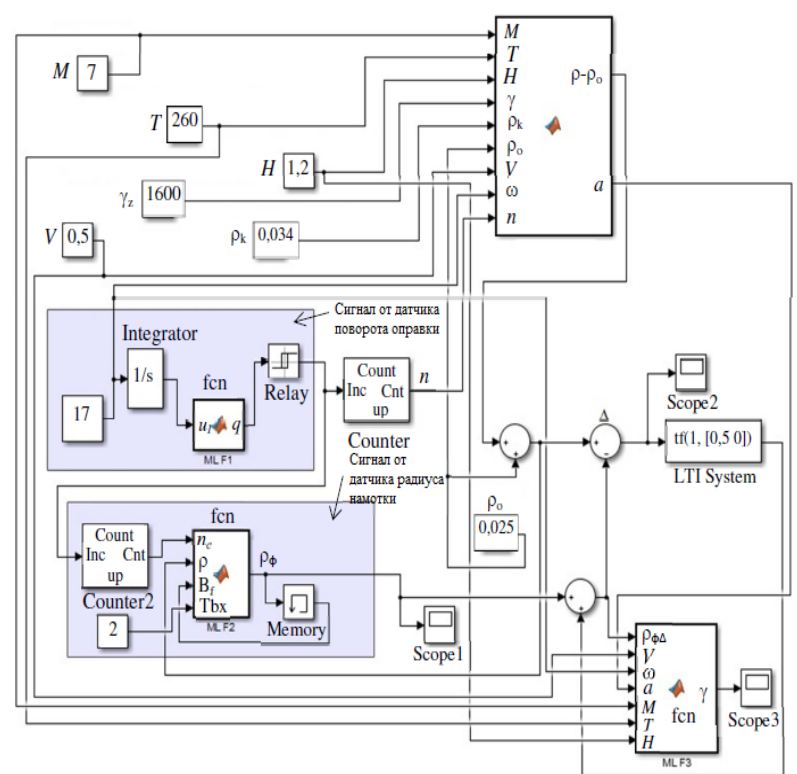

Figure 4. Dynamic model of the control process

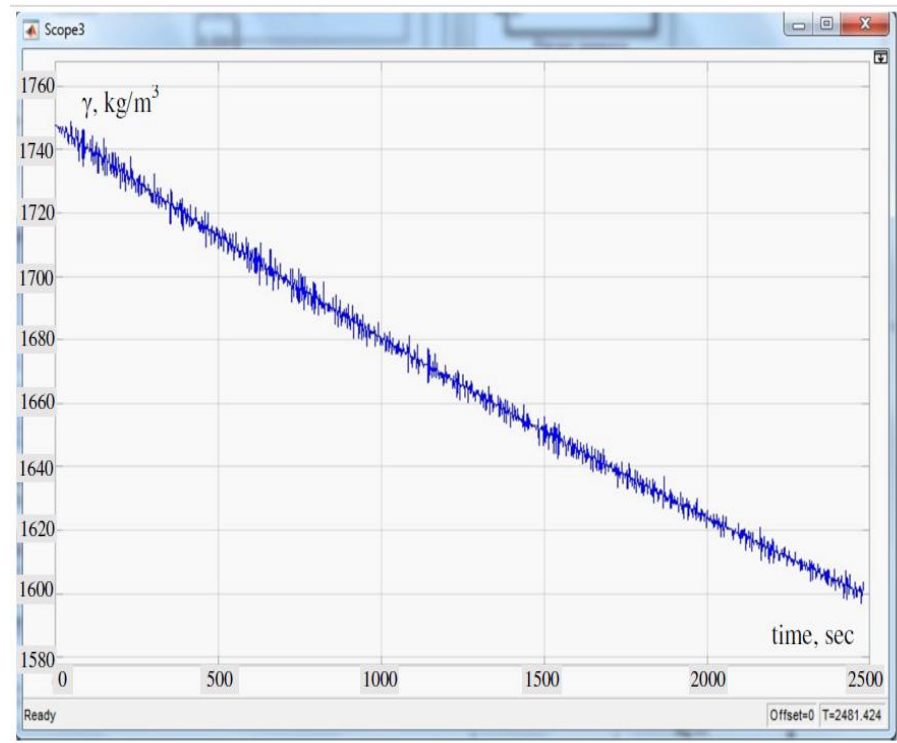

Figure 5. Changing the winding density during the winding process

\section{CONCLUSIONS}

A sufficiently effective solution is proposed for controlling the density of the composite winding in order to stabilize the stressstrain state of cylindrical composite products by changing the radial increment of the winding layers and preventing the action of random factors. Its effectiveness is due to the principle of direct action of the sealing member on the wound body and the low cost of the control system in contrast to other proposals in this area. based on the authors experience in robotics and field [Zhigailov, 2016], [Musalimov, 2018], future studies are planned to implement more mechatronics aspects to improve the composite windings solution.

\section{ACKNOWLEDGMENTS}

The result was obtained through the financial support of the Ministry of Education, Youth and Sports of the Czech Republic and the European Union (European Structural and Investment Funds - Operational Programme Research, Development and Education) in the frames of the project "Modular platform for autonomous chassis of specialized electric vehicles for freight 
and equipment transportation", Reg. CZ.02.1.01/0.0/0.0/16_025/0007293.

\section{REFERENCES}

[Hashimoto 2016] Hashimoto H. Intelligent winding machine of plastic films for preventing both wrinkles and slippages, Modern Mechanical Engineering, 6, 2016, 20-31. http://dx.doi.org/10.4236/mme.2016.61003.

[Hashimoto 2010] Hashimoto H., et al. Optimum winding tension and nip-load into wound webs for protecting wrinkle and slippage, JSME Journal of Advanced Mechanical Design, Systems, and Manufacturing, 4, 214-225. http://dx.doi.org/10.1299/jamdsm.4.214.

[Komkov 2012] M. Komkov, V. Tarasov and A. Borodulin. Spiral winding of ends of composite shells with cylindrical and conical shapes, Herald of the Bauman Moscow State Technical University. Series Mechanical Engineering, N 4, pp. 78-85, 2012.

[Kutin 2018] Kutin A., Musalimov V.M., and Polyakov A.S, Design of composite winding and control of the process of its formation, Proceedings of higher educational institutions. Instrumentation, vol. 61, no. 2, 2018, pp. 160-166.

[Kutin 2004] Kutin A. Estimation of the error in determining the length of a textile material, Technology of the textile industry. 2004. No. 2. pp. 117-118.

[Li 2015] Z. Li. Tension control system design of a filament winding structure based on fuzzy neural network // Engineering Review, Vol. 35, Issue 1, 9-17, 2015.

[Musalimov 2016] Musalimov V.M., Monakhov Yu.S., Kutin A.Yu., Solovieva G.A. Simulation of the process of winding threads on a rigid cylinder, Proceedings of higher educational institutions. Instrumentation. 2016. No. 8. C. 657-663.

[Musalimov, 2018] Musalimov, V., Aryassov, G., Zhigailov, S., Rõbak, D., Penkov, I. Motion imitation of the human pelvic and hip joints. 14th International Conference Mechatronic Systems and Materials, MSM 2018, Zakopane, Poland, 4 - 6 June 2018.

[Obraztsov 1986] Obraztsov I.F. Bulychev L.A., Vasiliev V.V. et al. Structural mechanics of aircraft: a textbook for aviation specialties of universities, Mechanical engineering, 1986. $536 \mathrm{c}$.

[Quanjin 2018] Ma Quanjin, M.R.M.Rejab, M.S.Idris, Nallapaneni Manoj Kumar, M.N.M. Merzuki. Robotic filament winding technique (RFWT) in industrial application: A review of state of the art and future perspectives, International Research Journal of Engineering and Technology (IRJET), Vol. 5, Issue 12, 16681675, Dec. 2018.

[Saad Mutasher 2012] Saad Mutasher, Nazim Mir Nasari, Lee Chai Lin. Small-scale filament winding machine for producing fiber composite products, Journal of Engineering Science and Technology Vol. 7, No. 2(2012) $156-168$.

[Shengle 2009] Ren Shengle, Lai Yi-nan, Wang Yong-zhangand Lu Hua. A new fiber winding precision tension control system, Proceedings of the IEEE International Conference on Automation and Logistics Shenyang, China August 2009.

[Sorrentino 2017] L. Sorrentino, M. Marchetti, C. Bellini, A. Delfini, F. Del Sette. Manufacture of high performance isogrid structure by Robotic Filament Winding, Composite Structures, vol. 164, pp. 43-50, 2017.

[Srivastava 2016] Srivastava S, Shariqul H. A brief theory on latest trend of filament winding machine, International Journal of Advanced Engineering Research and Science (IJAERS), Vol-3, Issue 4, 33-38, April- 2016.

[Thwaits 1977] Thwaits J. J. The elastic deformation of a rod with helical anisotropy, Int. J. Mech. - 1977. Vol. 19, №3. - P. 161169.

[Weifeng 2013] Zhong Weifeng, Yang Honghong, Li Huazhong, Xuliazhong. Control system design of robotized filament winding for elbow pipe, 2nd International Conference on Measurement, Information and Control, 2013.

[Zhang 2017] Zhang H, Tang H, Shi Y. Precision tension control technology of composite fiber tape winding molding // Journal of Thermoplastic Composite Materials, Vol. 31, Issue 7, 925-945, September 2017.

[Zhigailov, 2016] S. Zhigailov, V. Musalimov, G. Aryassov, I. Penkov. Modelling and Simulation of Human Lower-Limb Motion. International Review on Modelling and Simulations (I.RE.MO.S.), Vol.9, N2, ISSN 1974-9821, 114-121, 2016.

\section{CONTACT:}

Ing.Mgr Reza Moezzi.

Institute for Nanomaterials, Advanced Technologies and Innovation, Technical University of Liberec Studentska 2, Liberec 461 17, Czech Republic +420 48535 3920, Reza.Moezzi@tul.cz, https://cxi.tul.cz/ 